\title{
Understanding the distinctive elastic constants in an oxadiazole bent-core nematic liquid crystal
}

\author{
S. Kaur, ${ }^{1}$ J. Addis, ${ }^{1}$ C. Greco, ${ }^{2}$ A. Ferrarini, ${ }^{2}$ V. Görtz, ${ }^{3}$ J. W. Goodby, ${ }^{3}$ and H. F. Gleeson ${ }^{1, *}$ \\ ${ }^{1}$ School of Physics and Astronomy, University of Manchester, Manchester M13 9PL, United Kingdom \\ ${ }^{2}$ Department of Chemical Sciences, University of Padua, I-35131 Padua, Italy \\ ${ }^{3}$ Department of Chemistry, University of York, York YO10 5DD, United Kingdom
}

(Received 12 July 2012; published 5 October 2012)

\begin{abstract}
The splay and bend elastic constants of the bent-core oxadiazole material [C5-Ph-ODBP-Ph-OC12] have been investigated as a function of temperature across the nematic phase. The bend constant $K_{33}$ is found to take values of $\sim 3.0 \mathrm{pN}$ and to be almost temperature independent, whereas, the splay constant $K_{11}$ increases monotonically from $\sim 3.5 \mathrm{pN}$ close to the isotropic phase transition to values of $\sim 9 \mathrm{pN}$ deep in the nematic phase. No pretransitional divergence is observed in either $K_{11}$ or $K_{33}$ at temperatures approaching the underlying phase. This behavior of the elastic constants is distinct from that observed in rodlike liquid crystal systems but appears to share characteristics with the few other bent-core nematic systems studied to date. We discuss the interdependence of the elastic constants, the birefringence, and the order parameter to allow a comparison of the observed behavior with theory. We show that calculations of the elastic constants via molecular-field theory and atomistic modeling are in excellent qualitative as well as good quantitative (within $2 \mathrm{pN}$ ) agreement with the measurements across the temperature range, offering a deeper understanding of the elasticity in bent-core nematic materials than has been, hitherto, available.
\end{abstract}

DOI: 10.1103/PhysRevE.86.041703

PACS number(s): 61.30.Eb, 61.30.Gd, 78.15.+e

\section{INTRODUCTION}

The nematic liquid crystal (NLC) phase is the least ordered but most commonly used liquid crystal state, forming the basis of almost all commercial liquid crystal devices. Nematic liquid crystals are characterized by long-range orientational order, and the combination of their fluidity, optical anisotropy, and responsiveness to electric fields makes them attractive electro-optic materials. Most nematic phases are formed from either rodlike (calamitic) or disklike (discotic) molecules, but recently, systems formed by molecules with a bent-core structure have emerged as an exciting new class of nematogens as they offer the potential for polar order (ferronematics) and biaxiality. Indeed, even nonpolar uniaxial NLC phases formed from bent-core materials have been shown to exhibit remarkable physical properties including giant flexoelectricity [1,2], Kerr effect [3], unusual elastic constants and viscosities [4], and anomalous electroconvection [5-7].

Many bent-core mesogens exhibit highly ordered phases, characterized by both orientational and positional orders, and nematic phases tend to be observed only in systems with relatively low molecular bend $\left(\gtrsim 140^{\circ}\right)$. The oxadiazoles, which were, at first, considered inappropriate for the design of rodlike liquid crystalline materials due to their $\sim 135^{\circ}$ bend angle, were found to exhibit a nematic phase if the position of the central core unit was shifted from the middle to the periphery of the molecule [8]. Despite the high transition temperatures, the oxadiazoles became the most extensively investigated bent-core mesogens as nematic biaxiality was first claimed by Madsen et al. and Acharya et al. in these materials $[9,10]$. Later, however, the presence of a biaxial nematic phase was questioned by Vanakaras et al., suggesting that the phase could consist of uniaxially distributed biaxial clusters [11]. Francescangeli et al. further explained the splitting of small

*Helen.gleeson@manchester.ac.uk angle $\mathrm{x}$-ray diffraction patterns, taken to be a signature of a biaxial nematic phase, as the presence of skewed smectic fluctuations (cybotactic groups) [12].

The behavior and performance of NLC devices is dominated by the viscoelastic properties of the material; the elastic constants influence the threshold voltage for switching, whereas, the elastic constant ratio dictates the steepness of the electro-optic response and, therefore, limits to the complexity of the device. More fundamentally, there is considerable interest in studying the behavior of elastic constants because of the insight they offer in areas, such as testing continuum theory, understanding director distortion in devices, and providing insight into the microscopic structure of the ordered state and order parameter fluctuations [13]. The behavior of the elastic constants associated with the splay $K_{11}$, twist $K_{22}$, and bend $K_{33}$ deformations of the director in calamitic liquid crystals is well understood, although interestingly, it has emerged over the past decade that materials that include bent-core nematics are atypical $[14,15]$. Dodge et al. showed that the bend elastic constant (measured both by Freedrickz transition and light scattering experiments) reduced to half the original value in a mixture of calamitic and bent-core benzoate molecules [15]. Anomalous temperature dependence of $K_{11}$ and $K_{33}$ has been observed in mixtures of calamitic and bent-core carboxylate molecules and has been explained through the mutual alignment of both types of molecules [16]. Indeed, most of the studies focus on mixtures of calamitic and bent-core structures [14-18] with just a few that consider pure bent-core nematic systems $[4,19,20]$. The few reports of the elastic properties of purely bent-core nematic materials to date find that $K_{11}>K_{33}$ (the converse is true for calamitic nematic materials). Sathyanarayana et al. demonstrate that the temperature dependence of the elastic constants in a bent-core material is very different from that observed in calamitic systems and relate their findings to a coupling of the bent molecular shape with the bend distortions of the 
(a)

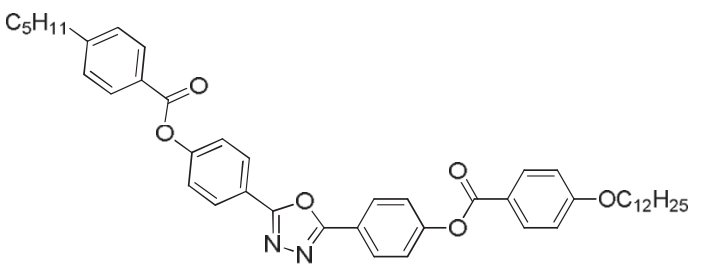

(b)

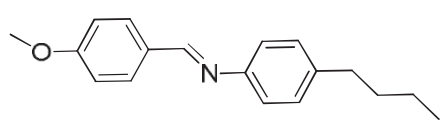

FIG. 1. Chemical structures of (a) C5-Ph-ODBP-Ph-OC12 and (b) MBBA.

director. Majumdar et al., however, suggest that nanoscale molecular clusters with tilted smectic correlations cause the softening of $K_{22}$ and $K_{33}$ relative to $K_{11}$. Salter et al. [21] also show that $K_{11}>K_{33}$ and find an unusually low ratio of $K_{33} / K_{11} \sim 0.5$ in a bent-core nematic material, although they find no anomalous flexoelectric behavior in contrast to Ref. [1]. It is clear that bent-core NLCs exhibit elastic properties that appear to be not readily explained by models developed for calamitic systems and that their unusual properties offer the potential for novel electro-optic devices. Indeed, Dozov [22] predicted pathological elasticity in bent-core materials resulting in the occurrence of new lower symmetry nematic phases, predictions that are now being applied to a few unusual bent-core nematic systems [23]. This paper offers important contributions to the understanding of elasticity in bent-core nematic systems as it reports the splay and bend elastic constants across the entire $\sim 60 \mathrm{~K}$ nematic range of an oxadiazole liquid crystal. This is the only system reported to date where the underlying phase is not a tilted smectic (smectic-C or SmC) phase but is, instead, the dark conglomerate (DC) phase. Importantly, we have carried out molecular-field atomistic calculations of the elastic constants for the molecule studied, and we compare these calculations to the experimental data.

\section{EXPERIMENTAL AND COMPUTATIONAL METHODS}

The bent-core mesogen studied is derived from a 1,3,4oxadiazole-biphenyl core (ODBP) and is shown in Fig. 1(a). The phase transitions of $\mathrm{C} 5$-Ph-ODBP-Ph-OC12 were measured to be isotropic $239.6{ }^{\circ} \mathrm{C} N 184.6{ }^{\circ} \mathrm{C} \mathrm{DC}$, where $N$ is the nematic phase and DC is the dark conglomerate phase; although the absolute values are higher than those quoted elsewhere [6,24], the nematic range is the same, and the difference is attributed to the absolute accuracy of hot stages at these high temperatures. All temperatures are, therefore, quoted with respect to the nematic to isotropic phase transition temperature $T_{\mathrm{NI}}$. This material is particularly interesting as studies using polarized Raman spectroscopy and small-angle $\mathrm{x}$-ray scattering [24] and electroconvection [6,7] have indicated anomalies in the low-temperature region of the nematic phase. Specifically, below $T-T_{\mathrm{NI}} \sim-30 \mathrm{~K}$, nonzero biaxial order parameters have been measured, and changes in the nature of electroconvection are also apparent. The x-ray data [24] show clear lobes in the small-angle scattering pattern. However, the angular peak separation of the lobes $\left(\sim 70^{\circ}\right)$ is temperature independent, and the perpendicular correlation length is only $\sim 1$ molecular length. The x-ray data [24] are, therefore, not consistent with the occurrence of cybotactic clusters [25]. Consequently, although the available data for C5$\mathrm{Ph}-\mathrm{ODBP}-\mathrm{Ph}-\mathrm{OC} 12$ do not confirm phase biaxiality, neither are the x-ray data consistent with a cybotactic nematic phase, and we note that the nature of the low-temperature nematic regime in $\mathrm{C} 5-\mathrm{Ph}-\mathrm{ODBP}-\mathrm{Ph}-\mathrm{OC} 12$ is, therefore, still an open question.

$\mathrm{C} 5-\mathrm{Ph}-\mathrm{ODBP}-\mathrm{Ph}-\mathrm{OC} 12$ exhibits negative dielectric anisotropy $\Delta \varepsilon$ across the entire nematic phase regime [7], and as a consequence, minor modifications are required for both the experimental geometry and the fitting programs that are more commonly used to measure elastic constants in materials with positive $\Delta \varepsilon$. We, therefore, checked our method using the negative calamitic nematic material $N$-p-methoxybenzylidene-p-butylaniline (MBBA), which has phase transitions: isotropic $42{ }^{\circ} \mathrm{C} N$, Fig. 1(b).

All measurements employ devices with glass substrates and a liquid crystal layer $\sim 5 \mu \mathrm{m}$ thick. The transparent (indium-tin-oxide) electrode area is $30 \mathrm{~mm}^{2}(20 \Omega / \square)$, and a high-temperature conducting glue (H21D, Epoxy Technology) is used to attach the wires to the electrode surfaces. The electrical properties of the devices were calibrated using air as a standard reference at all temperatures studied, and the devices were capillary filled at $190{ }^{\circ} \mathrm{C}$ for C5-Ph-ODBP-Ph-OC12 and at $\sim 30{ }^{\circ} \mathrm{C}$ for MBBA. Either planar or homeotropic alignment was required depending on the experiment carried out; the former was achieved using a high-temperature (SE130) polyimide layer, and the latter can be obtained using trichloroocta-decyl silane in heptane. Excellent monodomain alignment was confirmed by use of polarizing microscopy, although we note that there was no indication of any biaxial nematic regime via conoscopy in the homeotropic device. The devices were held in a Linkam THMS600 hot stage equipped with a TMS 93 controller, which allowed temperature control of the samples with a relative accuracy of $\pm 0.1{ }^{\circ} \mathrm{C}$.

The following subsection presents, in detail, the techniques employed in the measurement of birefringence and elastic constants followed by the calculation details of atomistic modeling.

\section{A. Birefringence measurements}

The birefringence measurements are undertaken via an analysis of the reflection spectra from planar aligned devices using the Berreman methodology [26]. This technique is particularly useful for accessing refractive indices at high temperatures where Abbé refractometers cannot be used. The details of both the apparatus used and the fitting procedure are given in Ref. [27]; the fitting program implements an optical model for the propagation of electromagnetic waves in media consisting of isotropic and anisotropic layers. Reflection spectra are first taken for the empty device, allowing the thickness to be measured with an accuracy of $\pm 0.02 \mu \mathrm{m}$, before filling at $200{ }^{\circ} \mathrm{C}$. The ordinary $n_{o}$ and extraordinary $n_{e}$ refractive indices are determined from spectra obtained with the nematic director oriented perpendicular and parallel 
to the incident light polarization, respectively. The average refractive index $\bar{n}=\sqrt{\left(2 n_{o}^{2}+n_{e}^{2}\right) / 3}$ and the birefringence $\Delta n=n_{e}-n_{o}$ may then be calculated with an accuracy of \pm 0.001 . All values of refractive index are quoted for light of wavelength $589 \mathrm{~nm}$.

\section{B. Measurement of the elastic constants $K_{11}$ and $K_{33}$}

The elastic constants are determined from the variation in the material dielectric constant through the electric-field induced Freedrickz transition. Homeotropic alignment is required since $\mathrm{C} 5$ - $\mathrm{Ph}-\mathrm{ODBP}-\mathrm{Ph}-\mathrm{OC} 12$ has negative $\Delta \varepsilon$ across the whole nematic regime, and in this geometry, the director experiences an electric torque on the application of the electric field perpendicular to the plane of the device. Obtaining homeotropic alignment for bent-core nematic materials is a particular challenge, although we have obtained excellent results for this material using trichloro-octa-decyl silane in heptane. The apparatus includes an Agilent Precision LCR Meter E4980A, which measures the dielectric permittivity across the frequency range $20 \mathrm{~Hz}-2 \mathrm{MHz}$ using a $0.05 \mathrm{~V}_{\mathrm{ac}}$ probe voltage where necessary in the presence of an ac electric field of up to $20 \mathrm{~V}$. The parallel component of the dielectric permittivity $\varepsilon_{\|}$is observed at fields below the Freedrickz threshold, whereas, at increasing field strength, above the transition, the director begins to align perpendicular to the field, thus, allowing the perpendicular component of the dielectric permittivity $\varepsilon_{\perp}$ to be deduced. The bend elastic modulus $K_{33}$ is directly related to the threshold voltage $V_{\text {th }}$ by

$$
V_{\mathrm{th}}=\pi\left(K_{33} / \varepsilon_{0} \Delta \varepsilon\right)^{1 / 2} .
$$

The splay elastic constant $K_{11}$ influences the steepness of the variation in capacitance above the threshold and can be deduced using a computer fitting program, which employs the following equation:

$$
\frac{V}{V_{\mathrm{th}}}=2 \pi^{-1}\left(1+\gamma \sin ^{2} \theta_{m}\right)^{1 / 2} \int_{0}^{\pi / 2}\left(\frac{F_{k}}{F_{\zeta} F_{\gamma}}\right)^{1 / 2} d \theta,
$$

where $F_{k}(\theta)=1+k \sin ^{2} \theta_{m}, F_{\zeta}(\theta)=1-\sin ^{2} \theta_{m}, F_{\gamma}(\theta)=$ $1+\gamma \sin ^{2} \theta_{m}, \quad k=\left(K_{11} / K_{33}\right)-1, \quad \gamma=\left(\varepsilon_{\perp} / \varepsilon_{\|}\right)-1$, and $\theta_{m}$ is the director angle in the center of the liquid crystal layer [28]. This methodology allows the elastic constants to be deduced with an accuracy of around $5 \%$. We note that no account has been taken of any possible contribution due to flexoelectricity in the sample.

\section{Calculation of elastic constants and definition of the molecular geometry}

The elastic constants of C5-Ph-ODBP-Ph-OC12 were calculated assuming a uniaxial nematic phase, according to the surface interaction model, wherein, the orientational mean field experienced by a molecule in the nematic phase $U^{\text {or }}$ is parametrized according to the anisotropy of the molecular surface. It is expressed as

$$
U^{\mathrm{or}}(\Omega)=\varepsilon k_{B} T \int_{S} d S P_{2}(\boldsymbol{n} \cdot \boldsymbol{s})
$$

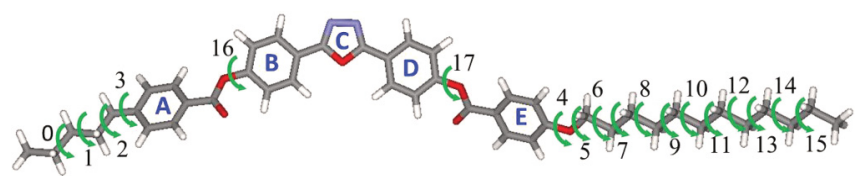

FIG. 2. (Color online) Molecular structure of C5-Ph-ODBP-PhOC12 with arrows showing the bonds that were allowed to rotate in Monte Carlo sampling of conformations. The corresponding dihedrals are denoted as $\chi_{i}(i=0-15)$.

where $\Omega$ are the Euler angles, which define the molecular orientation, $k_{B}$ is the Boltzmann constant, $T$ is the temperature, $P_{2}$ is the second Legendre polynomial, $\boldsymbol{n}$ and $\boldsymbol{s}$ are unit vectors parallel to the director and perpendicular to the surface element $d S$, respectively. The integral is over the molecular surface $S$, and $\varepsilon$ is a parameter that specifies the orienting strength. It is related to the reduced temperature and the molecular order parameters. In this framework, molecular expressions for the elastic constants are obtained as linear combinations of Cartesian tensor components, each defined as an integral over the molecular surface [29].

$\mathrm{C} 5-\mathrm{Ph}-\mathrm{ODBP}-\mathrm{Ph}-\mathrm{OC} 12$ is a highly flexible molecule, and the prediction of the elastic constants and orientational order parameters requires calculation of an average over all the conformational degrees of freedom of the core and the lateral wings. The rotational isomeric state (RIS) approximation was used [30], according to which, only the conformations corresponding to the minima of the torsional potential (the so called conformers) are considered. RIS conformers were collected by Monte Carlo (MC) sampling. The bonds of C5-Ph-ODBP-Ph-OC12 that were allowed to rotate are shown in Fig. 2; the corresponding dihedrals are denoted as $\chi_{i}(i=$ $0-15)$. At each MC move, a random number of bonds was allowed to jump from one to another state, and then, the new conformer was accepted on the basis of its torsional energy according to the Metropolis criterion [31].

In our calculations, planar geometry of the central three ring moiety was assumed in agreement with the results of density-functional theory calculations for 2,5-diphenyl1,3,4-oxadiazole reported in Ref. [32]; the torsional potential reported there for the bond between phenyl and oxadiazole exhibits two minima for planar structures, separated by barriers of about $30 \mathrm{~kJ} / \mathrm{mol}$ corresponding to the perpendicular arrangement. Planar or nearly planar geometry of 2,5-diphenyl1,3,4-oxadiazole was also found in crystals [33]. The geometry choices for the phenyl benzoate groups are less obvious since the potential energy surface of this group has been the object of several investigations with some differences in the results [34-36]. There is general agreement about the preference for the planar geometry of the benzoate unit due to the presence of high energy barriers, which oppose the rotation of the bond between the aromatic carbon $\left(\mathrm{C}_{\mathrm{ar}}\right)$ and the $\mathrm{COO}$ moiety $\left(\mathrm{C}_{\mathrm{ar}}-\mathrm{COO}\right)$. Thus, in our MC calculations, the benzoate unit was frozen in the planar geometry. Some discrepancies exist in the literature on the torsional potential for the $\mathrm{C}_{\mathrm{ar}}-\mathrm{C}_{\mathrm{ar}}-\mathrm{O}-\mathrm{CO}$ dihedral. Energy barriers at $0^{\circ}$ and $180^{\circ}$ (planar arrangement) are found, ranging from about $3 \mathrm{~kJ} \mathrm{~mol}^{-1}$ to more than $10 \mathrm{~kJ}$ $\mathrm{mol}^{-1}$. A smaller, in several cases, even insignificant barrier is found for the perpendicular arrangement of the phenyl rings, whose height strongly depends on the level of the theory. Thus, 
despite some uncertainty about the details, all calculations point to a wide distribution of the $\mathrm{C}_{\mathrm{ar}}-\mathrm{C}_{\mathrm{ar}}-\mathrm{O}-\mathrm{CO}$ dihedral in the ranges of $\pm\left(30^{\circ}-150^{\circ}\right)$. For the sake of simplicity, in our calculations, we assumed for $\chi_{16}$ and for $\chi_{17}$ two values $\pm 90^{\circ}$ in the middle of their highest probability region. The torsional potential for the $\mathrm{C}_{\mathrm{ar}}-\mathrm{CH}_{2}$ bond exhibits two equivalent minima, corresponding to the geometry with the first $\mathrm{CH}_{2}-\mathrm{CH}_{2}$ bond of the alkyl chain lying on a plane perpendicular to the aromatic plane [37]; therefore, we assumed two equivalent states with $\chi_{3}$ equal to $\pm 90^{\circ}$. Also, the torsional potential for the $\mathrm{C}_{\mathrm{ar}}-\mathrm{O}$ bond has two equivalent minima, but in this case, they correspond to the $\mathrm{O}-\mathrm{CH}_{2}$ bond lying on the same plane of the aromatic ring [37]; the values $\chi_{4}=0^{\circ}$ and $\chi_{4}=180^{\circ}$ were used in our calculations. For the $\mathrm{CH}_{2}-\mathrm{CH}_{2}$ and the $\mathrm{O}-\mathrm{CH}_{2}$ bonds in the lateral wings, three states were taken: the trans conformation (dihedral equal to $180^{\circ}$ ) and the gauche + , gauche - pair $\left( \pm 65^{\circ}\right)$. These three states are not energetically equivalent, and this was taken into account for sampling the conformer space according to the Metropolis criterion. The torsional energy of the $J$ conformer was calculated as

$$
\begin{aligned}
V^{J}= & \sum_{i=0, \ldots, 2,5, \ldots, 15}\left(\delta_{i, g+}^{J}+\delta_{i, g-}^{J}\right) \Delta V_{i} \\
& +\sum_{i=0,1,5, \ldots, 14}\left(\delta_{i, g+}^{J} \delta_{i+1, g+}^{J}+\delta_{i, g-}^{J} \delta_{i+1, g-}^{J}\right) \Delta V_{i, i+1},
\end{aligned}
$$

where the index $i$ indicates the bond and $\delta$ is the Kronecker symbol $\left(\delta_{i, g \pm}^{J}=1\right.$ if, in the $J$ conformer, the $i$ bond is in the $g_{ \pm}$state). The first sum accounts for the energy difference between the gauche and the trans states; $\Delta V_{i}=2.2 \mathrm{~kJ} \mathrm{~mol}^{-1}$ was assumed for all the chain dihedrals with the exception of $\chi_{6}$ for which $\Delta V_{6}=-2.85 \mathrm{~kJ} \mathrm{~mol}^{-1}$ was used [37]. The second sum in Eq. (4) introduces the energy stabilization associated with the presence of adjacent gauche + gauche + (or gauche - gauche - ) pairs, and $\Delta V_{i, i+1}=-0.75 \mathrm{~kJ} \mathrm{~mol}^{-1}$ was assumed [37]. In sampling the conformers of $\mathrm{C} 5-\mathrm{Ph}$ ODBP-Ph-OC12, structures having pairs of atoms closer than a cutoff distance equal to $0.82 \sigma$, where $\sigma$ is the sum of their van der Waals radii, were discarded. Van der Waals radii equal to $0.185 \mathrm{~nm}(\mathrm{C}), 0.15 \mathrm{~nm}(\mathrm{~N}$ and $\mathrm{O})$, and $0.1 \mathrm{~nm}(\mathrm{H})$ [38] were assumed. In this way, sterically hindered conformations were rejected, including those with adjacent gauche + gauche(or gauche - gauche + ) pairs in the hydrocarbon chains.

For each conformer, the molecular surface was generated by the fast molecular surface calculation library (MSMS) [39], assuming a rolling sphere radius equal to $0.3 \mathrm{~nm}$ and density of vertices equal to $5 \AA^{-2}$. The same van der Waals radii used for the cutoff distance were assumed for this purpose.

\section{RESULTS}

\section{A. Birefringence and order parameter of C5-Ph-ODBP-Ph-OC12}

Figure 3 shows the variation in birefringence in the nematic phase of C5-Ph-ODBP-Ph-OC12 as a function of reduced temperature. The birefringence increases monotonically with decreasing temperature as expected and as observed for calamitic systems. The inset to Fig. 3 shows explicitly the ordinary, extraordinary, and average refractive indices in the nematic phase as well as the isotropic refractive index. The

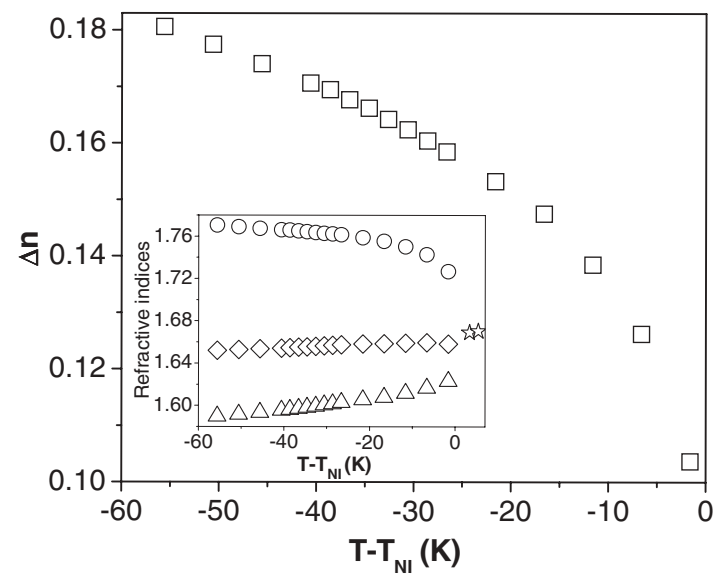

FIG. 3. Birefringence $\Delta n$ as a function of reduced temperature $T-T_{\mathrm{NI}}$ of C5-Ph-ODBP-Ph-OC12. The inset shows the temperature variation in the refractive indices, open circles: $n_{e}$; open triangles: $n_{o}$; and open rhombus: its average in the nematic and that in the open star: isotropic phase of C5-Ph-ODBP-Ph-OC12.

average refractive index is slightly lower than the isotropic phase value and, although a similarly small difference is often observed in calamitic materials, it is more common for the isotropic refractive index to take lower values than the mean value. The only comparable data published for bent-core liquid crystals is for an oxazole-derived system measured at infrared wavelengths $(1.5 \mu \mathrm{m})$ where there is no difference reported between the mean value of the refractive index in the nematic phase and the isotropic value [40].

The birefringence of $\mathrm{C} 5-\mathrm{Ph}-\mathrm{ODBP}-\mathrm{Ph}-\mathrm{OC} 12$ varies from $\sim 0.1$ to $\sim 0.18$ across the temperature range and is around a factor of 2 higher (near $T_{\mathrm{NI}}$ ) than that reported by Sathyanarayana et al. for a different bent-core material [19]. In the case of mixtures of rodlike and bent-core molecules, $\Delta n$ has generally been found to decrease with the increasing concentration of bent-core molecules $[16,18]$. The orientational order parameter $S$ can be deduced from the refractive index data following the well-known method of Haller [41]:

$$
S=\left(\frac{n_{e}^{2}-n_{o}^{2}}{\bar{n}^{2}-1}\right)\left(\frac{\bar{\alpha}}{\Delta \alpha}\right),
$$

where $\bar{n}^{2}=\frac{1}{3}\left(n_{e}^{2}+2 n_{o}^{2}\right), \bar{\alpha}=\frac{1}{3}\left(\alpha_{\|}+2 \alpha_{\perp}\right)$, and $\Delta \alpha=\alpha_{\|}-$ $\alpha_{\perp} . \alpha_{\|}$and $\alpha_{\perp}$ give the polarizability of individual molecules [42]. The temperature dependence of $S$ can be written as

$$
S=\left(1-\frac{T}{T^{*}}\right)^{\beta},
$$

where $T^{*}$ and $\beta$ are adjustable fitting parameters and $T$ is the temperature measured in Kelvin. The fitting parameters $\left[T^{*}=512.82 \mathrm{~K},(\bar{\alpha} / \Delta \alpha)=1.89\right.$, and $\left.\beta=0.19\right]$ are deduced by equating Eqs. (5) and (6). Although our value of $\beta$ is much less than 0.5 , which is predicted by mean-field theory, it is in good agreement with measurements of many other systems; Haller observed 13 different liquid crystals that exhibit $\beta \sim 0.2 \pm 0.03$, whereas, recent papers by Sathyanarayana et al. and Kundu et al. find $\beta \sim 0.2$ for both calamitic materials and mixtures of calamitic and bent-core materials $[18,19,43]$. Figure 4 shows the order parameter as a function 


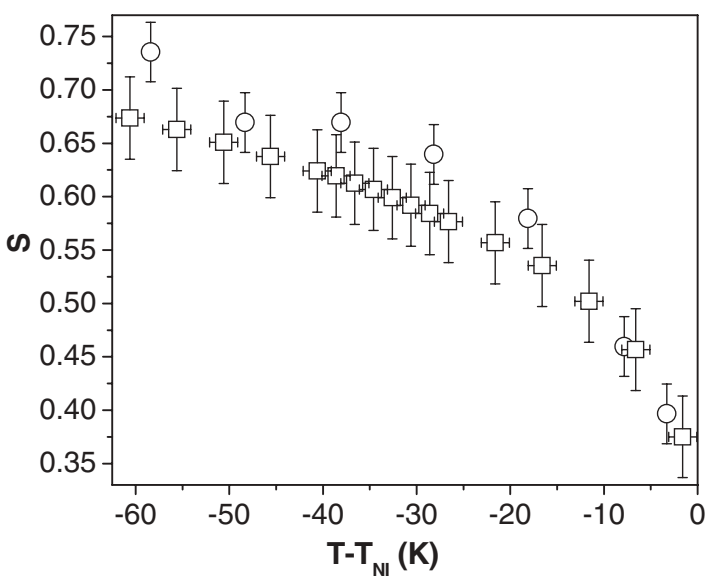

FIG. 4. Order parameter $S$ as a function of reduced temperature $T-T_{\mathrm{NI}}$ obtained from open squares: refractive index and open circles: Raman data.

of reduced temperature deduced from refractive index data together with the value obtained for the same material from Raman spectroscopy in which $P_{200}$ is equivalent to $S$ [24]. There is excellent agreement between these order parameter measurements as well as with those made on a different bent-core nematic material via NMR by Dong [44], Dong et al. [45], and Weissflog et al. [46]. Order parameters also deduced from refractive index data for a different material [19] are slightly lower than those in Fig. 4 but exhibit a similar temperature dependence.

\section{B. Elastic constants of C5-Ph-ODBP-Ph-OC12 determined experimentally}

First, the elastic constants of the calamitic liquid crystal MBBA are given in Table I. These data are within 5\% of those reported elsewhere [47], validating our methodology. The dielectric anisotropy of C5-Ph-ODBP-Ph-OC12 is found to decrease from -1.4 at $T-T_{\mathrm{NI}}=-8.7 \mathrm{~K}$ to -3.2 at $T-T_{\mathrm{NI}}=-56.7 \mathrm{~K}$. Figure 5 shows the splay $K_{11}$ and bend $K_{33}$ elastic constants of C5-Ph-ODBP-Ph-OC12 as a function of reduced temperature. $K_{11}$ increases monotonically with decreasing temperature, more than doubling over the temperature range, whereas, $K_{33}$ is almost temperature independent. Examining the bend elastic constant in more detail, it can be seen to increase slightly as the temperature decreases until below a reduced temperature of $T-T_{\mathrm{NI}} \sim-45 \mathrm{~K}$, a very

TABLE I. Measurement of $K_{11}$ and $K_{33}$ in the nematic phase of MBBA showing consistency with the reported values within $\pm 5 \%$ error.

\begin{tabular}{lcc}
\hline \hline$T-T_{\mathrm{NI}}(\mathrm{K})$ & $K_{11}(\mathrm{pN})$ & $K_{33}(\mathrm{pN})$ \\
\hline-18.5 & 6.2 & 7.4 \\
-15.5 & 5.0 & 7.0 \\
-12.5 & 5.0 & 6.2 \\
-9.5 & 4.1 & 5.6 \\
-6.5 & 4.1 & 4.7 \\
-3.5 & 3.1 & 3.7 \\
-0.5 & 2.0 & 2.0 \\
\hline \hline
\end{tabular}

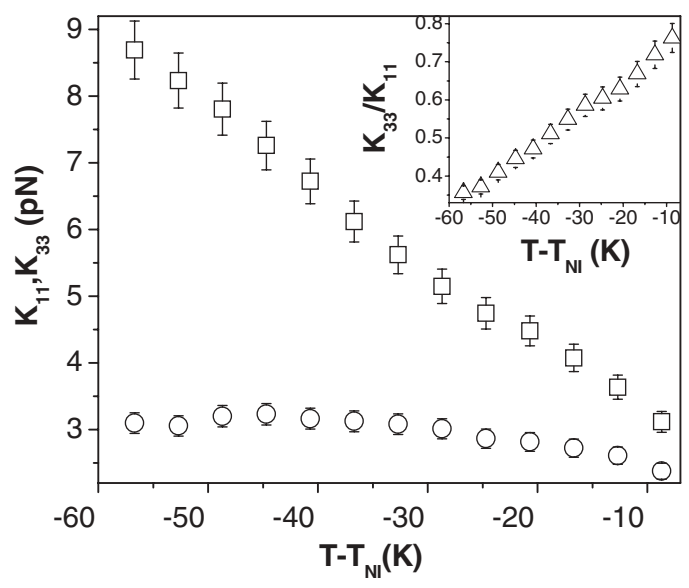

FIG. 5. Open squares: splay $K_{11}$ and open circles: bend $K_{33}$ elastic constants as a function of reduced temperature $T-T_{\mathrm{NI}}$ in the nematic phase. The inset shows the temperature variation in open triangles: $K_{33} / K_{11}$.

small decrease is suggested by the data. It is clear that there is no evidence of pretransitional divergence of either $K_{11}$ or $K_{33}$. Both Sathyanarayana et al. [19] and Tadapatri et al. [20] report elastic constants in bent-core materials with underlying $\mathrm{SmC}$ phases, both also describing an approximately linear temperature dependence of $K_{11}$, varying between $\sim 2-17$ and $\sim 2-28 \mathrm{pN}$, respectively. The behavior of $K_{33}$ appears to be quite different in each case, being almost temperature independent here, increasing continuously from $\sim 1$ to $5 \mathrm{pN}$ in Ref. [20], and showing a strong pretransitional divergence in Ref. [19]. The inset of Fig. 5 shows that the ratio of $K_{33} / K_{11}$ of C5-Ph-ODBP-Ph-OC12 decreases from 0.8 to 0.3 as the temperature decreases. Values as low as 0.52 have been observed in long-chain calamitic nematics [48], although it is more usual in calamitic systems that $K_{11}<K_{33}$.

Discussions of the temperature dependence of the elastic constants in bent-core nematic materials have employed a comparison of the data with the order parameter. As a simple interpretation of mean-field theory (discussed in more detail in the following section) predicts a dependence of the elastic constants on $S^{2}$, several authors have examined the relationship $K_{i i} \propto \Delta n^{x}$. In order to facilitate a comparison of our data with that in Refs. [19,20], Fig. 6 show plots of $K_{11}$ and $K_{33}$ (inset) as a function of birefringence $\Delta n$ and order parameter $S$. Using this approach, the data for $K_{11}$ appear to behave differently in the low- and high-temperature regimes, changing at $T-T_{\mathrm{NI}} \sim-30 \mathrm{~K}$; there is approximately a factor of 2 difference in the exponents associated with each region, which are $\sim 2.5$ and 4.5 for the high- and low-temperature regimes, respectively (a single exponent does not fit the data over the whole nematic regime). Interestingly, the change in behavior occurs at approximately the same temperature where anomalous behavior has been reported for this compound via Raman spectroscopy and electroconvection measurements [7,24]. The anomaly in $K_{11}$ observed in the high- and lowtemperature regimes is not clear from either the splay elastic constant or the order parameter data alone. In comparison, examining the relationship $K_{i i} \propto \Delta n^{x}$ has resulted in values of $x=2.9$ for the $K_{11}$ data of Sathyanarayana et al. [19] 


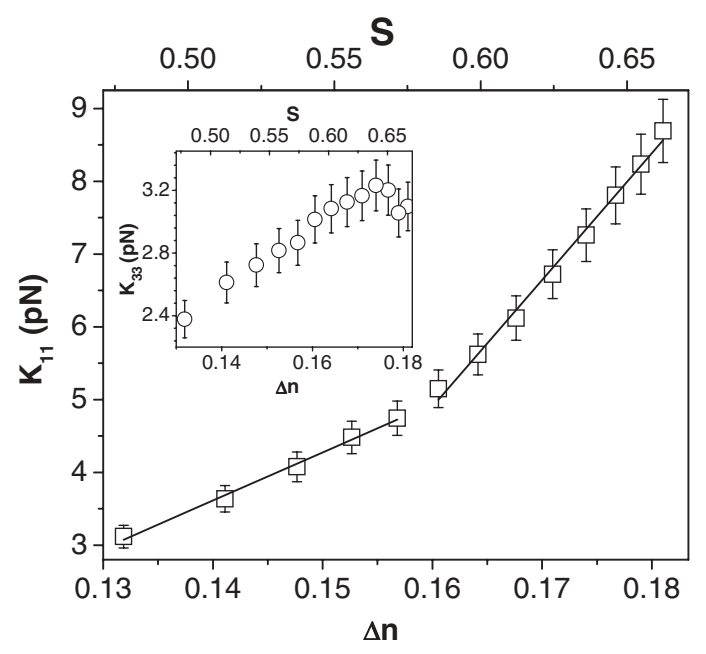

FIG. 6. Open squares: splay elastic constant $K_{11}$ as a function of bottom axis: birefringence $\Delta n$ and top axis: order parameter $S$ in the nematic regime. The inset shows the behavior of open circles: $K_{33}$ as a function of bottom axis: $\Delta n$ and top axis: $S$.

and values of $x=2.5$ for mixtures of rod and bent-core nematic materials [16]. The inset of Fig. 6 shows that the bend elastic constant $K_{33}$ exhibits an approximately linear dependence on $\Delta n$ until at $T-T_{\mathrm{NI}}=-44.7 \mathrm{~K}(\Delta n \approx 0.174)$ and, thereafter, decreases. Analogous results are observed in high concentration mixtures of bent-core molecules in 5CB where $K_{33}$ increases with increasing $\Delta n$ and attains a maximum at a certain $\Delta n$ value before decreasing [18]. Kundu $e t a l$. also observed a decrease in $K_{33}$ with decreasing temperature in a $17 \mathrm{M}$ concentration of bent-core molecules in $80 C B$ [16].

\section{DISCUSSION}

The behavior of the elastic moduli of C5-Ph-ODBP-PhOC12 can be summarized as follows:

(1) In common with other bent-core nematic materials $K_{11}>K_{33}$, differing from calamitic nematic systems.

(2) The values of both elastic constants are comparable to but slightly lower than in other bent-core systems.

(3) The birefringence takes values of around 0.18 , and the order parameters deduced from both refractive index data and Raman scattering give values between 0.35 and 0.70 , comparing well with order parameter data for other bent-core nematic materials.

(4) The temperature dependence of $K_{11}$ is analogous to other bent-core materials, increasing approximately linearly with reducing temperature. However, its relationship with the order parameter shows two distinct regimes with exponents of around 2.5 and 4.5 at high and low temperatures, respectively.

(5) The behavior of $K_{33}$ is quite distinct for this system; it is almost temperature independent, taking a maximum value of $\sim 3 \mathrm{pN}$. There is some evidence of a decrease in $K_{33}$ at low temperatures in the nematic regime, but there is no obvious pretransitional divergence.

The elastic constants clearly behave differently from calamitic systems, and although there are some similarities with other bent-core systems, there are also differences. In the subsequent sections, we first consider the elastic behavior in the context of existing physical models followed by computational investigation of our system.

\section{A. Examining existing physical models}

We organize the discussion to consider first the absence of critical divergence on approaching the underlying phase as this is perhaps the simplest result to understand. We then consider the relationship of the elastic constants with the order parameter and, finally, the values of the elastic constants and their ratios.

According to de Gennes [49] and Chen and Lubensky [50], all three nematic elastic constants diverge at the SmC-N phase transition. Findon and Gleeson [51] report that, for a calamitic nematic mixture, $K_{11}$ increases continuously from $\sim 4$ pN close to the nematic to isotropic transition to $\sim 30 \mathrm{pN}$ close to the SmC transition. Although there is some evidence of the variation becoming slightly more rapid close to the transition, there is no clear pretransitional behavior, and $K_{11}$ is continuous across the transition into the SmC phase where it takes values ranging from $\sim 30$ to $100 \mathrm{pN}$. The temperature dependence of $K_{11}$ reported here and by others for different bent-core materials [19-21] is in broad agreement, and it increases approximately linearly across the nematic phase range as the temperature reduces. On the other hand, Sathyanarayana $\mathrm{et} \mathrm{al}$. report a strong pretransitional divergence in $K_{33}$, observed neither by us nor in Refs. [20,21]. In both cases (Refs. [19] and [20]), the same general behavior might be expected as the underlying phase is SmC. However, in $\mathrm{C} 5-\mathrm{Ph}-\mathrm{ODBP}-\mathrm{Ph}-\mathrm{OC} 12$, there is a first-order transition to an underlying DC phase, and although the elastic behavior in that phase has not yet been considered either theoretically or experimentally, a first-order transition normally severely curtails pretransitional phenomena. Therefore, the lack of pretransitional divergence in the elastic constants reported here is as expected. Similar behavior might also be expected for the material in Ref. [21] as the underlying phase is a crystal phase, but measurements are reported only for temperatures $7 \mathrm{~K}$ below the isotropic phase transition, well away from the nematic to crystal transition.

We next consider the theoretical relationship between elastic constants and order parameter. Nehring and Saupe [52], Saupe [53], and Khoo and Wu [54] showed that a mean-field approach suggests that $K_{i i}$ are related to the order parameter $S$ by

$$
K_{i i}=C_{i i} V_{n}^{-7 / 3} S^{2},
$$

where $C_{i i}=\left(\frac{3 \mathrm{~A}^{0}}{2}\right)\left(\mathrm{Lm}^{-1} \gamma_{i i}^{-2}\right)^{1 / 3}$ and $C_{i i}$ are reduced elastic constants that depend on molecular properties; $V_{n}$ is the molar volume, $L$ is the molecular length, $m$ is the number of molecules in a steric unit, $A^{0}=1.3 \times 10^{-8} \mathrm{erg} \mathrm{cm}^{6}$, and $\gamma_{11}=\gamma_{22}=z / x$ and $\gamma_{33}=(x / z)^{2}$, where $x, y$, and $z$ are the average molecular lengths in directions across and along the molecular long axis. For calamitic materials with small alkyl chains, $C_{i i}$ are found to be temperature independent, but as the chain length increases, $C_{i i}$ have considerable temperature dependence, and to account for the decrease in $K_{33} / K_{11}$ with increasing chain length (discussed further below), the increase in $C_{11}$ is found to be more than for 

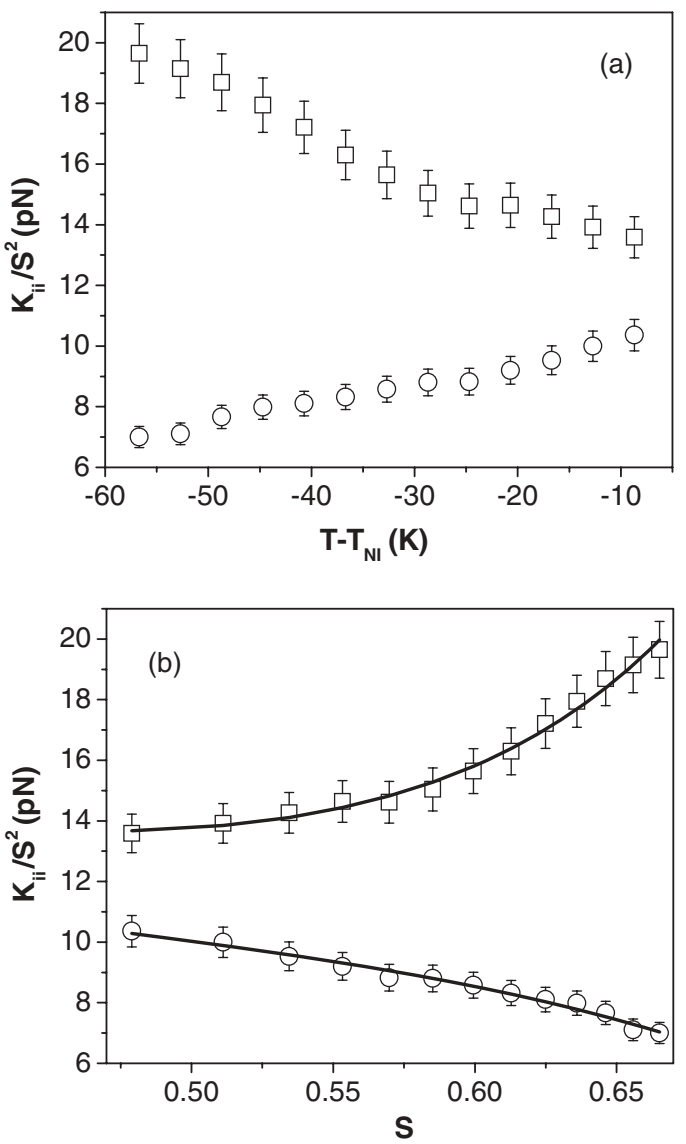

FIG. 7. $K_{i i} / S^{2}$ (where $i$ stands for 1 and 3 ) plotted as a function of (a) reduced temperature, $T-T_{\mathrm{NI}}$. (b) Order parameter $S$. Open squares denote $K_{11}$, and open circles denote $K_{33}$. Solid lines are a fit to Eq. (8).

$C_{33}$. Indeed, de Jeu and Claassen showed that $C_{33} / C_{11}$ is the square of the ratio of the correlation length parallel $\xi_{\|}$ and perpendicular $\xi_{\perp}$ to the director, respectively [55]. It is clear that the elastic constants are predicted to depend on $S^{2}$ only if the assumption of no significant temperature dependence of $C_{i i}$ is valid. The ratio $K_{i i} / S^{2}$ is shown as a function of reduced temperature in Fig. 7(a), illustrating the significant temperature dependence of $C_{i i}$ suggested using this approach. Given the importance of clustering in bent-core nematic systems, we consider whether this could be related to correlation lengths, following the approach of de Jeu with regard to the relationship between $C_{i i}$ and the correlation lengths. Data on the parallel and perpendicular correlation lengths are unavailable for our material, but taking results for another oxadiazole bent-core nematic liquid crystal [Fig. 2(b) of Ref. [56]], the value of $\left(\xi_{\|} / \xi_{\perp}\right)^{2}$ increases with decreasing temperature [at $T-T_{\mathrm{NI}}=-18 \mathrm{~K},\left(\xi_{\|} / \xi_{\perp}\right)^{2}=2.6$, which increases to 4.5 at $T-T_{\mathrm{NI}}=-113 \mathrm{~K}$ ]. In our system, $C_{33} / C_{11}$ (which is equivalent to $K_{33} / K_{11}$, inset to Fig. 5) decreases from $\sim 0.8$ close to $T_{\mathrm{NI}}$ to $\sim 0.35$ at $T-T_{\mathrm{NI}}=-60 \mathrm{~K}$, a trend that is completely opposite to that expected from clustering. Indeed, the perpendicular correlation length for $\mathrm{C} 5-\mathrm{Ph}-\mathrm{ODBP}-$ $\mathrm{Ph}-\mathrm{OC} 12$ is approximately temperature independent and is $\sim 43 \AA$ [24], meaning that, if clustering was behind the behavior of the elastic constants, the clusters would be characterized by a rather unexpected shape, tens of molecules wide and only one molecule long. Given this and the nature of our underlying phase transition, we suggest that an explanation other than clustering must be sought to explain the elastic behavior of this and potentially other bent-core nematic systems.

The second-order dependence of the elastic constants on the order parameter [Eq. (7)] follows the arguments presented by other authors who have studied elastic constants in bent-core nematic systems, and it is tempting to suggest that the anomalous behavior observed in the exponents (Fig. 6) in the two different temperature regimes could suggest the emergence of biaxiality. However, a more detailed and less commonly used mean-field treatment of the elastic constants by Berreman and Meiboom [57] indicates that the correct expression to use includes a third-order dependence given by

$$
\frac{K_{i i}}{S^{2}}=K_{i}^{(2)}+K_{i}^{(3)} S+K_{i}^{(4)}\left(\frac{S}{1-S}\right)^{2},
$$

where $K_{i}^{(2)-(4)}$ are fitting parameters (which could, in principle, be temperature dependent). The ratio $K_{i i} / S^{2}$ is shown as a function of order parameter $S$ in Fig. 7(b), and the solid lines show fits of Eq. (8) to our data assuming no temperature dependence in $K_{i}^{(2)-(4)}$ (measured in $\mathrm{pN}$ ), which are found to be $K_{1}^{(2)}=20.2 \pm 2.1, K_{1}^{(3)}=-19.2 \pm 4.6, K_{1}^{(4)}=$ $3.2 \pm 0.3, K_{3}^{(2)}=14.4 \pm 1.5, K_{3}^{(3)}=-7.5 \pm 3.3$, and $K_{3}^{(4)}=$ $-0.6 \pm 0.2$.It is clear that excellent fits are obtained, although only if the condition that $K_{11}$ and $K_{33}$ converge at $S=0$ [57] is relaxed.

We next consider the ratio $K_{33} / K_{11}$ in our bent-core nematic liquid crystal. It has been shown that there is a correlation between $K_{33} / K_{11}$ and $L / W$ where $L$ and $W$ are the length and breadth of the molecule [48,58]. For short-chain calamitic nematic systems, this naturally leads to $K_{33} / K_{11}>1$ as the molecular length is greater than the breadth. However, there are several systems where the ratio is modified. In the case of the homologous series of $p, p^{\prime}$-di- $n$-alkyl and $p, p^{\prime}$-di$n$-alkoxy azobenzenes, both $K_{11}$ and $K_{33}$ become greater with increasing chain length, whereas, the ratio $K_{33} / K_{11}$ decreases along the series [55] due to an increase in the number of conformations adopted by the flexible chain. In hockey-shaped molecules [59] as well as bent-core systems, the molecular shape and population of conformers can certainly lead to situations where $W_{\text {eff }}>L$ ( $W_{\text {eff }}$ is the effective width of the molecule). Finally, there are several papers that demonstrate that the lower-temperature regimes of bent-core systems include large cybotactic SmC or SmA clusters, which can explain many of the unique features of bent-core nematic systems and which could also lead to a situation where $W_{\text {eff }}>L$. Thus, there are several reasons why one might expect $K_{11}>K_{33}$ in bent-core systems, so it is not surprising that we and others $[16,19-21]$ report such a relationship. We note that, in the case of $\mathrm{C} 5-\mathrm{Ph}-\mathrm{ODBP}-\mathrm{Ph}-\mathrm{OC} 12$, there is no $\mathrm{X}$-ray evidence for pretransitional SmA or SmC clusters in the nematic regime [24], indeed, these would be unexpected given the first-order transition to a DC phase below the nematic phase.

In C5-Ph-ODBP-Ph-OC12, $K_{11}>K_{33}$ is common with other bent-core nematic systems but contrasts the behavior found in most calamitic liquid crystals. Interestingly, almost 40 years ago, Gruler [60] and Helfrich [61] each presented 
TABLE II. Influence of the Helfrich correction on the values of the bend elastic constant $K_{33}$ in selected bent-core nematic materials.

\begin{tabular}{lcccc}
\hline \hline Material/reference & $\beta(\mathrm{rad})$ & $T(\mathrm{~K})$ & $K_{33}(\mathrm{pN})$ & $\bar{K}_{33}(\mathrm{pN})$ \\
\hline C5-Ph-ODBP-Ph-OC12 & 0.7 & 475.0 & 3.0 & 3.4 \\
Reference [19] & 0.82 & 419.5 & 4.0 & 5.2 \\
Reference [4] & $\sim 1.0$ & 347 & 0.88 & 0.97 \\
\hline \hline
\end{tabular}

models that predicted a decrease in the bend elastic constant in the case of banana-shaped molecules. Helfrich employed phenomenological and molecular considerations to argue that

$$
K_{33}=\frac{\bar{K}_{33}}{1+\beta^{2} N_{0}^{-1 / 3}\left(\bar{K}_{33} / 2 k_{B} T\right)},
$$

where $\bar{K}_{33}$ is the bend elastic modulus without molecular adjustment to the strain, $K_{33}$ is the modulus adjusted for the bend structure, $\beta$ is the molecular curvature $\left(180^{\circ}-\theta\right.$ bend), and $N_{0}$ is the molecular density. The above formula does not incorporate an internal field correction, the elongated molecular shape, or the degree of order, which are taken into consideration by Gruler [60]. Gramsbergen and de Jeu reported a mere $5 \%$ adjustment of $\bar{K}_{33}$ for a bent-core thiadiazole compound using the above formula [62]. In C5-Ph-ODBP$\mathrm{Ph}-\mathrm{OC} 12, K_{33}$ is $\sim 3 \times 10^{-12} \mathrm{~N}$ (from Fig. 5), $\beta=0.7 \mathrm{rad}$, $N_{0}=10^{27} \mathrm{~m}^{-3}, k_{B} T$ is $655.78 \mathrm{~J}$, where $T=475 \mathrm{~K}$, and we get $\bar{K}_{33}=3.38 \times 10^{-12} \mathrm{~N}$, which is again a rather small change $(\sim 10 \%)$. Indeed, it seems that this adjustment cannot account for any of the values of $K_{33}$ reported; Table II summarizes the influence of this correction on selected materials.

Clearly, the formula suggested by Helfrich offers a much smaller shift in the bend elastic constant than would be needed to return to the situation for calamitic liquid crystals where $K_{11}<K_{33}$. However, Gruler presented a molecular viewpoint of the elastic theory and obtained a much more significant effective change of $10 \mathrm{pN}$ in $K_{33}$ for bent molecules [60]. This suggests that the inclusion of intrinsic molecular deformations in the calculations for bend elastic constants is important. Indeed, in summarizing the discussion so far, it is clear that the elastic behavior of C5-Ph-ODBP-Ph-OC12 and other bent-core nematic systems is significantly different from that of calamitic systems, and we next consider whether a more sophisticated approach based on mean-field theory, which makes use of atomistic modeling is more suited to understanding these systems.

Recent calculations of this kind by Cestari and Ferrarini on typical mesogenic systems show that the high rigidity of paraazoxyanisole compared with $4-n$-alkyl,-4'-cyanobiphenyls (5 and $8 \mathrm{CBs}$ ) in which contributions of various conformers (due to the slightly bent shape) are important, results in lower values of elastic constants [63]. The number of possible molecular conformers increases with the increase in length of the flexible chain, and the authors emphasized that the presence of a V-shaped structure has a marked effect in lowering the bend elastic constant. A recent case study on two mesogenic odd dimers with a certain degree of bend in the all-trans state conformation by Cestari et al. predicted a low bend elastic constant, which can even become negative at higher-order parameters [64] and could be a prerequisite to the twist-bend nematic phase [23]. Nearly a decade earlier, Dozov also proposed that the natural tendency of banana-shaped molecules to induce bend distortions could result in negative $K_{33}$ values [22]. The next section presents the computational modeling of $\mathrm{C} 5-\mathrm{Ph}-\mathrm{ODBP}-\mathrm{Ph}-\mathrm{OC} 12$ and demonstrates how the elastic constants behave in the case of a bent-core system.

\section{B. Calculation of the elastic constants of C5-Ph-ODBP-Ph-OC12}

C5-Ph-ODBP-Ph-OC12 can assume markedly different shapes as a consequence of rotations around the bonds highlighted in Fig. 2. In our calculations, this was taken into account by averaging over the conformations of the lateral wings and of the aromatic core as explained in Sec. IIC. Based on considerations of the relevant torsional potentials, two conformations were assumed for the core, which differ from each other for having the carbonyl groups on opposite sides (I) and on the same side (II) of the oxadiazole ring as shown in Fig. 8(a). In these conformations, the angle between the para axes of the $\mathrm{B}$ and $\mathrm{D}$ phenyls is equal to $138^{\circ}$, and the angle between the $\mathrm{A}$ and $\mathrm{E}$ phenyls takes the same value. The calculated splay and bend elastic constants are reported in Fig. 8(b) as a function of the orientational order parameter $S_{z z}$ ( $z$ is the axis passing through the carbon atoms of the oxadiazole ring). We can see that, in agreement with the experiment and in contrast to the usual behavior of rodlike calamitics, $K_{11}>K_{33}$ is predicted. Also, in line with what is found experimentally, the calculated $K_{11}$ steadily increases with increasing order (it increases slowly with ordering in
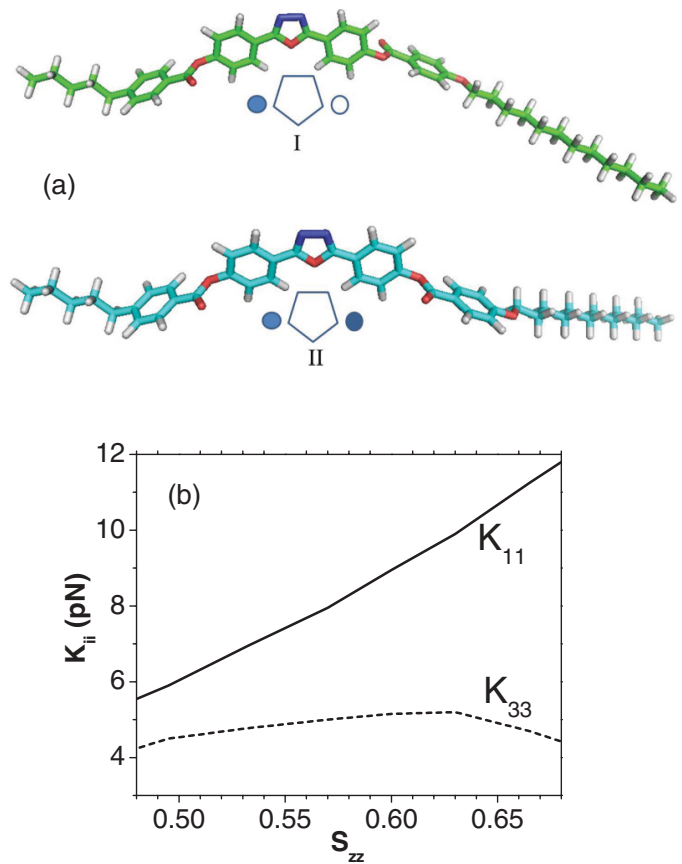

FIG. 8. (Color online) (a) Conformations of C5-Ph-ODBP-PhOC12 with all-trans hydrocarbon chains and different core configurations. The schematics show the orientation of the carbonyl groups: on opposite sides (I) and on the same side (II) of the oxadiazole ring. (b) Calculated elastic constants as a function of the order parameter for the axis passing through the carbon atoms of the oxadiazole ring. 

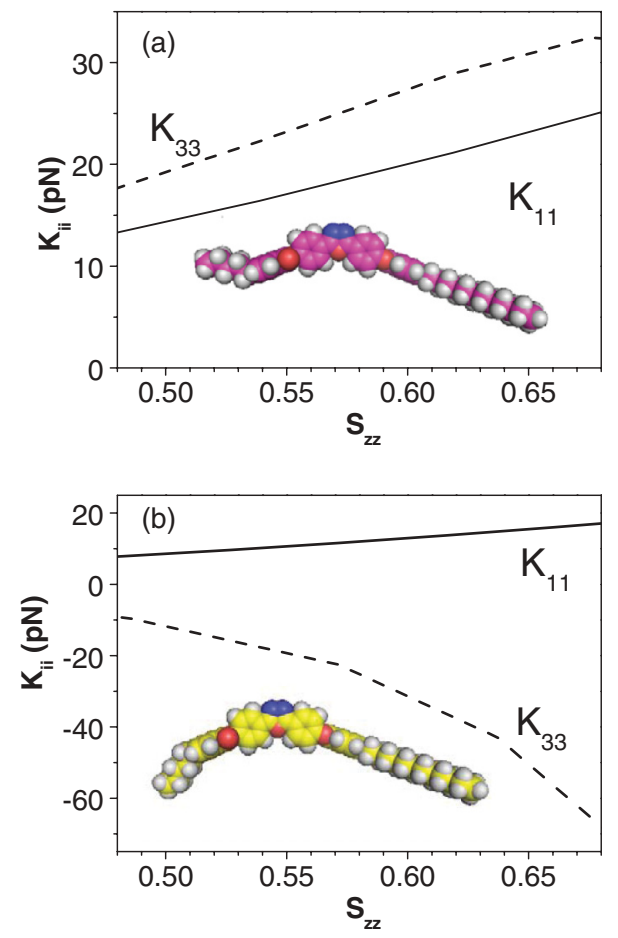

FIG. 9. (Color online) (a) and (b) Elastic constants calculated for the two single conformers (shown in the insets) as a function of the order parameter for the axis passing through the carbon atoms of the oxadiazole ring.

calamitic NLCs), whereas, $K_{33}$ remains nearly constant and tends to decrease at high ordering (it increases with order in calamitic NLCs). Both $K_{11}$ and $K_{33}$ are slightly higher than the experimental values, but strict quantitative agreement is probably beyond the present capability because of the detailed knowledge of the molecular geometry that is needed for this purpose.

To get an insight into the relationship between molecular structure and elastic constants, it can be useful to look at Figs. 9(a) and 9(b), which show the results of calculations for a couple of distinct conformers of C5-Ph-ODBP-Ph-OC12 (shown in the insets): They both have the core in the I configuration and the all-trans chains and only differ in the value of the $\chi_{3}$ dihedral, which is equal to $+90^{\circ}$ in one case and to $-90^{\circ}$ in the other. We can see that the rotation around a single bond, changing the curvature of the molecular structure, has dramatic effects on the bend elastic constant. For the less bent conformer, a more conventional behavior is predicted, $K_{33}>$ $K_{11}$ is found, and both elastic constants are large as expected for a large molecule, such as C5-Ph-ODBP-Ph-OC12. For the other conformer, $K_{11}$ remains large, but $K_{33}$ takes negative values, which would be incompatible with the existence of a uniform nematic phase because it implies a tendency for spontaneous bending of the director [22]. We can explain the unusual behavior of $K_{33}$ reported in Fig. 8(b) as the result of different contributions, which lead, on average, to small $K_{33}$ values with a weak temperature dependence: Elongated conformers, which would impart the nematic phase with high bending stiffness, are simultaneously present with bent conformers, which would promote bending of the mesophase director. At very high ordering, the negative contribution to
$K_{33}$ becomes huge and prevails. It was already shown that, even for conventional NLCs, the molecular curvature may have a strong influence on the bend elastic constant [63]. This effect is enhanced by the peculiar structure of bent-core NLCs. Other systems whose bend elasticity was found to be particularly sensitive to conformational changes are odd mesogenic dimers as mentioned earlier. For those systems, $K_{11}>K_{33}$ was again predicted, along with a nearly temperature independent $K_{33}$, decreasing at lower temperatures [64]. Such theoretical predictions were subsequently confirmed by the experiment [65].

\section{CONCLUSION}

In conclusion, splay and bend elastic constants along with birefringence and order parameter measurements of a bent-core oxadiazole NLC have been reported. The order parameter derived from the refractive indices is in excellent agreement with that obtained from Raman spectroscopy. The splay elastic constant is found to be higher than the bend elastic constant in common with other bent-core systems. It is interesting to note that no pretransitional behavior in either $K_{11}$ or $K_{33}$ is observed close to the transition to the underlying DC phase. Various existing models have been considered to explain the fact that $K_{11}>K_{33}$, but none could fully explain the behavior of splay and bend in the bent-core materials. The relationship between the elastic constants and the order parameter is found to be in excellent agreement with the equation suggested by Berreman and Meiboom [57], which includes third-order terms, provided that the condition of convergence of splay and bend at zero-order parameter is relaxed. Elastic constants were also determined from molecular-field theory and atomistic modeling, and notably good agreement was found with the experimental results. The value of $K_{11}$ is found to be almost independent of the molecular conformation, whereas, $K_{33}$ is highly geometry dependent, and in particular, it is extremely sensitive to changes in the molecular curvature. Importantly, the calculations show that what has been considered to be anomalous behavior of the elastic constants can, in fact, be predicted by molecularfield theories of nematics, provided that the bent molecular shape is suitably taken into account. An important distinctive feature evidenced by both our experimental results and the calculations is that $K_{33}$ can reduce to extremely low values well below the nematic to isotropic transition temperature, which is due to the difficulty in reconciling a bent molecular shape with a uniform director at high-order parameter values. This feature, which could be of interest for applications where low elastic constants are required, is also highly relevant to recent observations of splay-bend nematic phases in dimeric liquid crystals. Indeed, the low cost of bend deformations could well be important in the formation of domains of opposite chirality, recently observed in the nematic phase of bent-core bis-(phenyl)oxadiazole derivatives [66]. Finally, we note that this analysis of the splay and bend elastic constants of C5-Ph-ODBP-Ph-OC12 gives no insight into any possible biaxial nature of the nematic phase of this system, although it is clear that cybotactic clusters need not be considered to be present to explain the observed physical properties. 


\section{ACKNOWLEDGMENTS}

This work was supported by the Engineering and Physical Sciences Research Council under Project No. EP/G023093/1.
C.G. acknowledges the Ph.D. School of Materials Science and Engineering (University of Padova) and Merck Chemicals Ltd. for financial support. We thank D. Allender for useful discussions.
[1] J. Harden, B. Mbanga, N. Éber, K. Fodor-Csorba, S. Sprunt, J. T. Gleeson, and A. Jákli, Phys. Rev. Lett. 97, 157802 (2006).

[2] J. Harden, M. Chambers, R. Verduzco, P. Luchette, J. T. Gleeson, S. Sprunt, and A. Jákli, Appl. Phys. Lett. 96, 102907 (2010).

[3] S. Dhara, F. Araoka, M. Lee, K. Van Le, L. Guo, B. K. Sadashiva, K. Song, K. Ishikawa, and H. Takezoe, Phys. Rev. E 78, 050701 (2008).

[4] M. Majumdar, P. Salamon, A. Jákli, J. T. Gleeson, and S. Sprunt, Phys. Rev. E 83, 031701 (2011).

[5] D. Wiant, J. T. Gleeson, N. Éber, K. Fodor-Csorba, A. Jákli, and T. Tóth-Katona, Phys. Rev. E 72, 041712 (2005).

[6] Y. Xiang, J. W. Goodby, V. Görtz, and H. F. Gleeson, Appl. Phys. Lett. 94, 193507 (2009).

[7] S. Kaur, A. Belaissaoui, J. W. Goodby, V. Görtz, and H. F. Gleeson, Phys. Rev. E 83, 041704 (2011).

[8] D. Girdziunaite, C. Tschierske, E. Novotna, H. Kresse, and A. Hetzheim, Liq. Cryst. 10, 397 (1991).

[9] L. A. Madsen, T. J. Dingemans, M. Nakata, and E. T. Samulski, Phys. Rev. Lett. 92, 145505 (2004).

[10] B. R. Acharya, A. Primak, and S. Kumar, Phys. Rev. Lett. 92, 145506 (2004).

[11] A. G. Vanakaras and D. J. Photinos, J. Chem. Phys. 128, 154512 (2008).

[12] O. Francescangeli, F. Vita, C. Ferrero, T. Dingemans, and E. T. Samulski, Soft Matter 7, 895 (2011).

[13] P. G. de Gennes, The Physics of Liquid Crystals (Clarendon, Oxford, 1974).

[14] M. R. Dodge, C. Rosenblatt, R. G. Petschek, M. E. Neubert, and M. E. Walsh, Phys. Rev. E 62, 5056 (2000).

[15] M. R. Dodge, R. G. Petschek, C. Rosenblatt, M. E. Neubert, and M. E. Walsh, Phys. Rev. E 68, 031703 (2003).

[16] B. Kundu, R. Pratibha, and N. Madhusudana, Phys. Rev. Lett. 99, 247802 (2007)

[17] P. Sathyanarayana, B. K. Sadashiva, and S. Dhara, Soft Matter 7, 8556 (2011)

[18] P. Sathyanarayana, V. S. R. Jampani, M. Skarabot, I. Musevic, K. V. Le, H. Takezoe, and S. Dhara, Phys. Rev. E 85, 011702 (2012).

[19] P. Sathyanarayana, M. Mathew, Q. Li, V. S. S. Sastry, B. Kundu, K. V. Le, H. Takezoe, and S. Dhara, Phys. Rev. E 81, 010702 (2010).

[20] P. Tadapatri, U. S. Hiremath, C. V. Yelamaggad, and K. S. Krishnamurthy, J. Phys. Chem. B 114, 1745 (2010).

[21] P. S. Salter, C. Tschierske, S. J. Elston, and E. P. Raynes, Phys. Rev. E 84, 031708 (2011).

[22] I. Dozov, Europhys. Lett. 56, 247 (2001).

[23] M. Cestari et al., Phys. Rev. E 84, 031704 (2011).

[24] C. D. Southern, P. D. Brimicombe, S. D. Siemianowski, S. Jaradat, N. Roberts, V. Görtz, J. W. Goodby, and H. F. Gleeson, Europhys. Lett. 82, 56001 (2008).
[25] C. Keith, A. Lehmann, U. Baumeister, M. Prehm, and C. Tschierske, Soft Matter 6, 1704 (2010).

[26] D. W. Berreman, J. Opt. Soc. Am. 62, 502 (1972).

[27] H. G. Yoon, N. W. Roberts, and H. F. Gleeson, Liq. Cryst. 33, 503 (2006).

[28] L. M. Blinov and V. G. Chigrinov, Electrooptic Effects in Liquid Crystal Materials (Springer-Verlag, New York, 1994).

[29] M. Cestari, A. Bosco, and A. Ferrarini, J. Chem. Phys. 131, 054104 (2009).

[30] P. W. Flory, Statistical Mechanics of Chain Molecules (Wiley-Interscience, New York, 1969).

[31] N. Metropolis, A. W. Rosenbluth, M. N. Rosenbluth, A. H. Teller, and E. Teller, J. Chem. Phys. 21, 1087 (1953).

[32] J. P. Laguno, Ph.D Thesis, Durham University, 2007.

[33] I. Orgzall, F. Emmerling, B. Schulz, and O. Franco, J. Phys.: Condens. Matter 20, 295206 (2008).

[34] R. Wrzalik, K. Merkel, and A. Kocot, J. Mol. Model. 9, 248 (2003).

[35] G. Cinacchi and G. Prampolini, J. Phys. Chem. A 109, 6290 (2005).

[36] S. Kawauchi, S.-W. Choi, K. Fukuda, K. Kishikawa, J. Watanabe, and H. Takezoe, Chem. Lett. 36, 750 (2007).

[37] M. Cestari, Ph.D Thesis, University of Padova, 2009.

[38] D. R. Lide, Handbook of Chemsitry and Physics (CRC, Boca Raton, FL, 1996)

[39] M. F. Sanner, A. J. Olson, and J.-C. Spehner, Biopolymers 38 , 305 (1996).

[40] J. Olivares, S. Stojadinovic, T. Dingemans, S. Sprunt, and A. Jákli, Phys. Rev. E 68, 041704 (2003).

[41] I. Haller, Prog. Solid State Chem. 10, 103 (1975).

[42] M. F. Vuks, Opt. Spectrosc. 20, 644 (1966).

[43] B. Kundu, R. Pratibha, and N. V. Madhusudana, Eur. Phys. J. E 31, 145 (2010).

[44] R. Y. Dong, J. Phys. Chem. B 113, 1933 (2009),

[45] R. Y. Dong, K. Fodor-Csorba, J. Xu, V. Domenici, G. Prampolini, and C. A. Veracini, J. Phys. Chem. B 108, 7694 (2004).

[46] W. Weissflog, S. Sokolowski, H. Dehne, B. Das, S. Grande, M. W. Schröder, A. Eremin, S. Diele, G. Pelzl, and H. Kresse, Liq. Cryst. 31, 923 (2004).

[47] W. H. De Jeu, W. A. P. Claassen, and A. M. J. Spruijt, Mol. Cryst. Liq. Cryst. 37, 269 (1976).

[48] W. H. de Jeu, Physical Properties of Liquid Crystalline Materials (Gordon and Breach Science, New York/London/Paris, 1980).

[49] P. G. de Gennes, Mol. Cryst. Liq. Cryst. 21, 49 (1973).

[50] J.-H. Chen and T. C. Lubensky, Phys. Rev. A 14, 1202 (1976).

[51] A. Findon and H. F. Gleeson, Ferroelectrics 277, 35 (2002).

[52] J. Nehring and A. Saupe, J. Chem. Phys. 56, 5527(1972).

[53] A. Saupe, Z. Naturforsch. 15, 815 (1960).

[54] I.-C. Khoo and S.-T. Wu, Optics and Nonlinear Optics of Liquid Crystals (World Scientific, Singapore, 1993), Vol. 1. 
[55] W. H. de Jeu and W. A. P. Claassen, J. Chem. Phys. 67, 3705 (1977).

[56] O. Francescangeli et al., Adv. Funct. Mater. 19, 2592 (2009).

[57] D. W. Berreman and S. Meiboom, Phys. Rev. A 30, 1955 (1984).

[58] G. Vertogen and W. H. de Jeu, Thermotropic Liquid Crystals, Fundamentals, Springer Series in Chemical Physics, Vol. 45 (Springer-Verlag, Berlin, 1988).

[59] P. Sathyanarayana, S. Radhika, B. K. Sadashiva, and S. Dhara, Soft Matter 8, 2322 (2012).

[60] H. Gruler, J. Chem. Phys. 61, 5408 (1974).
[61] W. Helfrich, Mol. Cryst. Liq. Cryst. 26, 1 (1974).

[62] E. F. Gramsbergen and W. H. de Jeu, Phys. Lett. A 97, 199 (1983).

[63] M. Cestari and A. Ferrarini, Soft Matter 5, 3879 (2009).

[64] M. Cestari, E. Frezza, A. Ferrarini, and G. R. Luckhurst, J. Mater. Chem. 21, 12303 (2011).

[65] K. L. Atkinson, S. M. Morris, F. Castles, M. M. Qasim, D. J. Gardiner, and H. J. Coles, Phys. Rev. E 85, 012701 (2012).

[66] V. Görtz, C. Southern, N. W. Roberts, H. F. Gleeson, and J. W. Goodby, Soft Matter 5, 463 (2009). 